\title{
AMCP Partnership Forum: Biosimilars-policy, practice, and postmarketing surveillance to support treatment and coverage decisions
}

\section{SUMMARY}

With the dual goals of identifying key actions that can support the further development and use of biosimilars in the United States and providing consistent and accurate messages about the value of biosimilars, AMCP held a virtual multidisciplinary stakeholder forum December 15-16, 2020. The participants, including payers, pharmacists, integrated delivery system leaders, health economists and analysts, academicians, patient advocates, pharmaceutical manufacturers, and other key decision makers, spent the 2 days (1) identifying challenges with biosimilar adoption within the US health care system; (2) determining clear and unbiased scientific messaging to support broader acceptance of biologics as valid therapeutic options and to facilitate faster time to biosimilar adoption; and (3) discussing needs and opportunities related to real-world evidence to help with adoption of biosimilars.

Participants identified various challenges, including approvalrelated nuances and other regulatory considerations; general information about biosimilars and lack of consistent, positive messaging; clinical and administrative barriers; and economic complexities and variation. They also highlighted areas of opportunity, such as demystifying information and developing strong, positive messaging around biologics and biosimilars, and improving confidence in biosimilars by using tools such as education and realworld evidence, organizational strategies to ease biosimilar adoption, and updates to legislation and regulation to reduce barriers related to biosimilars.

\section{Forum participants}

Christina Barrington, RPh, PharmD, Vice President, Pharmacy Programs, Priority Health; Diana Brixner, RPh, PhD, FAMCP, Executive Director, Pharmacotherapy Outcomes Research Center, University of Utah; Mary Jo Carden, JD, Head of Policy, Sandoz; Jack Cox, Senior Director, Nova Nordisk Inc.; Joe Eggler, Senior Director, Market Access Account Management, Fresenius Kabi; Erika Emerson, Executive Director, Diabetes Leadership Council; Alison Falb, JD, Regulatory Counsel, FDA; Anna Hyde, Vice President, Advocacy and Access, Arthritis Foundation; Jim Kenney, RPh, MBA, President, JTKENNEY, LLC; Troy Koch, PharmD, MBA, Account Medical Lead, Takeda Pharmaceuticals; April Kunze, PharmD, Senior Director, Clinical Formulary Development and Trend Management Strategy, Prime Therapeutics; Paul Lakomski, BS, RPh, MBA, Pharmacy Services Manager, Aetna/CVS; Jeffrey Larson, RPh, MS, MBA, Clinical Director, CVS Health; Molly Billstein Leber, PharmD, BCPS, FASHP, Associate Director, Drug Use Policy and Formulary Management, Yale New Haven Health; Hannah Lynch, MPS, Associate Director, Federal Government Relations and Health Policy, National Psoriasis Foundation; George Mayzell, MD, MBA, President, Empowered Healthcare; Dorothy McCabe, PhD, FCP, Executive Director, CDMA, Specialty Care, Boehringer Ingelheim Pharmaceuticals, Inc.; Corey McEwen, PharmD, MS, Director, Oncology Pharmacy Services, Massachusetts General Hospital; Ann McNamara, PharmD, Director of Clinical Development, Fairview Specialty Pharmacy; Therese Mulvey, MD, FASCO, Director, Quality Safety and Value, MGH Cancer Center; Farhana Naz, BS, Senior Director, Strategy, Fresenius Kabi; Sonia Oskouei, PharmD, BCMAS, DPLA, Vice
President, Biosimilars, Cardinal Health; Gary Owens, MD, President, Gary Owens Associates; Chad Pettit, MBA, Executive Director, Marketing, Amgen; Ronald Piervincenzi, PhD, Chief Executive Officer, United States Pharmacopeia (USP); Laura Pizzi, PharmD, MPH, Director and Professor, Health Outcomes, Policy, and Economics (HOPE) Program, Rutgers University; Jim Rebello, Vice President, Formulary Strategy, Magellan Rx Management; Carly Rodriguez, PharmD, FAMCP, Pharmacy Director, Clinical Innovation, Moda Health; Elizabeth Sampsel, PharmD, MBA, BCPS, Clinical Program Manager, Medlmpact Healthcare Systems Inc.; Marissa Schlaifer, MS, RPh, Vice President, Policy and Regulatory Affairs, OptumRx; Christine Simmon, JD, Senior Vice President, Policy and Strategic Alliances, and Executive Director, Biosimilars Council, Association for Accessible Medicines (AAM); Eva Temkin, JD, Acting Director for Policy, Office of Therapeutic Biologics and Biosimilars, Center for Drug Evaluation and Research, FDA; Jaap Venema, PhD, Executive Vice President and Chief Science Officer, USP; Randy Vogenberg, PhD, RPh, Principal and Board Chair, Institute for Integrated Healthcare (IIH) and EmployerProvider Interface Council (EPIC); and Erin Wright, PharmD, BCPS, Director, Field Pharmacy, Premier, Inc.

\section{CORRESPONDENCE:}

Vyishali Dharbhamalla, PharmD, AMCP, 703.684.2633; vdharbhamalla@amcp.org

J Manag Care Spec Pharm 2021;27(10):1503-08

Copyright (C2021, Academy of Managed Care Pharmacy. All rights reserved. 
Biological products comprise a diverse category of products that includes therapeutic proteins, monoclonal antibodies, and vaccines. ${ }^{1}$ They are generally large, complex molecules produced through biotechnology in a living system such as a microorganism, plant cell, or animal cell. The nature of biological products, including the inherent variations that can result from the manufacturing process, can present challenges in characterizing and manufacturing these products that often do not exist in the development of small-molecule drugs. ${ }^{1}$

For example, generic versions of brand name smallmolecule drugs can be approved by the US Food and Drug Administration (FDA) on the basis of a demonstration of bioequivalence via an abbreviated new drug application. ${ }^{2}$ It is referred to as abbreviated because, unlike for a new drug application for an innovative product, preclinical (animal) and clinical (human) data generally are not required in order to establish the drug's safety and effectiveness. ${ }^{2}$ Availability of generic medications is a vital part of the US health care system, as they represent $90 \%$ of all prescriptions and saved \$265 billion in 2017 alone. $^{3}$

Biosimilars are also versions of FDA-approved brand name drugs-called reference products-and have the potential to generate considerable cost savings in health care; however, given the aforementioned complexity and variability inherent to biologic products, a new abbreviated approval pathway based not just on bioequivalence was necessary for biosimilars. ${ }^{1}$ This pathway was created with the 2009 Biologics Price Competition and Innovation Act, which requires a biosimilar to demonstrate high similarity to its reference product; minor differences are acceptable in clinically inactive components, but there can be no clinically meaningful differences between the biosimilar and the reference product in terms of safety and effectiveness. ${ }^{1,4}$

Although this abbreviated pathway means that a biosimilar manufacturer is not required to demonstrate safety and effectiveness as with a reference product, reducing the need for extensive clinical trials, more data are generally required in order to establish biosimilarity than bioequivalence..$^{5}$ These data usually include some preclinical and clinical data. ${ }^{5}$

Despite this established pathway, uncertainties and resistance remain around the use of biosimilars. For example, in 2020 the AMCP Foundation conducted a survey on this topic, building on work done with a 2018 survey. Both surveys indicate the same 3 top barriers to biosimilar adoption-state laws/regulations for substitution and interchangeability, pricing and contracting issues, and prescribers' concerns about safety and efficacy-but as a barrier, state laws/regulations for substitution and interchangeability moved from third in 2018 to first in 2020, indicating its increased importance., ${ }^{6,7}$ The 2020 survey also found that among respondents involved in managed care, pharmacy benefit management (PBM), and specialty pharmacy, the percentage of those who indicated that they strongly agree that switching from a reference product to a biosimilar is considered safe and effective increased to $50 \%$ from $32 \%$ in 2018; however, those who responded as uncertain also increased slightly, from $8 \%$ in 2018 to $12 \%$ in 2020.

Given these and other data, and given the dual goals of identifying key actions that can support the further development and use of biosimilars in the United States and providing consistent and accurate messages about the value of biosimilars, AMCP held a virtual multidisciplinary stakeholder forum December 15-16, 2020. The participants, including payers, pharmacists, integrated delivery system leaders, health economists and analysts, academicians, patient advocates, pharmaceutical manufacturers, and other key decision makers, spent the 2 days (1) identifying challenges with biosimilar adoption within the US health care system; (2) determining clear and unbiased scientific messaging to support broader acceptance of biologics as valid therapeutic options and to facilitate faster time to biosimilar adoption; and (3) discussing needs and opportunities related to real-world evidence (RWE) to help with biosimilar adoption.

\section{Challenges Associated With Biosimilar Adoption in the United States}

Various factors cause the biosimilar landscape to be highly complex and diversified. Some of these factors were emphasized during the forum discussion, including nuances related to product approvals, confusion around terminology, differences in economics and incentives across various stakeholders, unique perspectives within various physician clinical specialties, complex electronic medical record systems and order sets, and individual patient needs that depend on the patient's disease state and whether they are treatment naive or established on a biological therapy. All of these factors present an overall challenge from which many other challenges flow.

Participants often highlighted approval-related nuances and other regulatory considerations as a considerable challenge to biosimilar adoption in the United States. In particular, the "biosimilar" and "interchangeable" designations were considered to be causing confusion, as the concept of interchangeability is novel in the United States and not part of the European experience of biosimilars, and to date market experience with interchangeable products 


\section{TABLE 1 AMCP Pre-Partnership Forum Survey Responses ( $N=22$ Respondents)}

Biosimilar product statements

\begin{tabular}{|c|c|c|c|c|c}
\multirow{3}{*}{$\begin{array}{c}\text { Mean } \\
\text { strength }^{\mathrm{a}}\end{array}$} & \multicolumn{5}{|c}{ Audience $^{\mathrm{b}}$} \\
\cline { 2 - 6 } & Patient & HCP & Payer & Manufacturer & Policy \\
\hline
\end{tabular}

Biosimilars are as safe and effective as the original biologic; both are rigorously and thoroughly evaluated by the FDA before approval.

Biosimilars are approved after rigorous scientific evaluation by regulatory authorities.

You can expect the same safety and effectiveness from a biosimilar during the course of treatment as you would from the reference product.

The same quality manufacturing standards that apply to the original biologic also apply to the biosimilar.

Supporting a competitive marketplace for biosimilars is essential for improving patient access to necessary medications and reducing costs.

Biosimilars enhance competition among biological medicines, providing more treatment alternatives for patients and clinicians.

Biosimilars have been used safely for many years.

Biosimilars and generics are versions of previously FDA-approved medications and may offer more affordable treatment options to patients.

An interchangeable biosimilar is expected to produce the same clinical result as the reference product in any given patient.

As part of the assessment process, biosimilars must demonstrate a high similarity to an already approved originator biological medicine.

Globally, regulators have confidence in the rigor of the scientific review and approval process for biosimilars.

The interchangeability designation is not superior to biosimilarity designation, and all biosimilars approved by the FDA are safe and effective.

Biosimilars are biological medicines with proven pharmaceutical quality.

Biosimilars have been increasingly used in clinical practice in most countries.

A full clinical development program is not necessary when extensive laboratory testing has demonstrated that the biosimilar is highly similar to the originator.

a Message strength scale: excellent $=5$, good $=4$, fair $=3$, poor $=2$, very poor $=1$.

${ }^{b} T$ he audience scale indicates percentage of responses: $++++, \geq 90 \%,+++, 75-89 \%,++, 50-74 \%,+,<50 \%$.

$F D A=U S$ Food and Drug Administration; $\mathrm{HCP}=$ health care provider.

is lacking. Additionally, biosimilars may not be approved for the same indications as their reference product, and different biosimilars for the same reference product may be approved for different indications, making it difficult to compare products and thus to prefer one product over another in many circumstances. Differences in state mandates regarding substitution and interchangeability, delayed availability of products once approved, and lack of differential Medicare reimbursement or similar policies to promote biosimilar utilization were also offered as examples of such challenges.

Ongoing challenges also exist with available information about biosimilars and a lack of consistent, positive messaging-a point that was underscored by the results of the survey participants were asked to complete before the forum (Table 1). Participants especially noted difficulty in communicating the concept of interchangeability given its uniqueness to the US biosimilar market. Also, in the United States, recent events have caused some members of the public to mistrust science in general and the drug approval process in particular. Participants recognized that this challenge may need to be overcome in order to help patients understand the positive benefits of biosimilars.

Forum participants also discussed a number of potential clinical and administrative barriers. Of particular note is that perspectives on biosimilars may vary among physician specialties, for example, rheumatology, gastroenterology, oncology; this variance may be related to the early 
hesitance of some organizations to embrace biosimilars in their guidelines or position statements, or to the nature of various conditions. For instance, most rheumatology patients receive long-term, chronic treatment, resulting in fewer patients who are new to therapy and thus more need to switch patients to another drug in order increase biosimilar utilization; oncology patients, on the other hand, often receive shorter-term therapy, meaning more patients are new to therapy, which is, in general, more conducive to increasing biosimilar utilization. Another suggested point of contrast between rheumatology and oncology was that oncologists might be more used to rapid evolution of evidence and changes to treatment paradigms, and so might be more comfortable prescribing biosimilars. Also, for many prescribers, because of requisites related to setting up electronic medical records and order sets in various systems, particularly when each patient might be associated with different payers and formularies, it can be difficult to determine the most appropriate biosimilar that will provide the right treatment at the right cost for a given patient, especially when the biosimilar is one drug in a multidrug regimen.

Economic complexities and variation were another challenge area identified by participants. Some of the existing complexities with health plans or PBMs, including contracting arrangements, may reduce or remove the anticipated cost savings of a biosimilar versus the reference biologic. Also, there may not be opportunities to enter into contracting arrangements for all categories of drugs in which biosimilars are available, and thus health plan strategies may differ depending on drug category. These economic complexities also may be true when considering implementing formulary changes or policies requiring biosimilar use in conditions for which the affected population includes more treatment-naive patients-more so than for conditions for which more patients are subject to drug switching, as with the rheumatology and oncology examples above. Switching inherently creates more impact on patients and providers, which necessitates greater input of resources by the health plan or PBM. Prescriber-specific economic challenges noted by participants might also be influenced by health plan/PBM decisions; these included no difference in reimbursement to providers between a biosimilar and its reference product and difficulty in managing inventory for providers who use the buy-and-bill model, have multiple patients, and potentially use multiple preferred biosimilar products.

Participants also acknowledged several patient considerations related to economics. Some reference products, for instance, may offer more robust patient support programs, making them a more attractive option from the patient perspective than a new-to-market biosimilar that does not offer any patient support. Certain patients, such as some of those with cancer, may be less concerned about the cost of therapy, which could lead to a lack of an incentive to choose a biosimilar. Lack of cost transparency may also play a role, given that most currently available biosimilars are covered under the medical benefit and are not subject to formulary tiering.

\section{Opportunities to Increase Biosimilar Adoption in the United States}

Participants considered demystifying information and providing strong, positive messaging around biologics and biosimilars as key opportunities to increase biosimilar adoption in the United States. Simplifying the language used to describe approved biosimilars was one proposed example and included adopting phrases such as as safe and effective as and therapeutically equivalent to rather than biosimilar and interchangeable. Additionally, currently used quality statements could benefit from the addition of comparative examples that use existing market experience, and continued postmarket surveillance and RWE generation could provide outcomes data to assist in message development. Amplifying the resources provided by the FDA and others was also suggested.

Specifically for clinicians and decision makers, participants believed it was important to improve messages around safety and efficacy, to use available outcomes data from RWE, and to emphasize economic benefits in a competitive marketplace, which includes biosimilars, and the implications for the overall cost and affordability of medications and their use within the health care system. Clinicians could consider speaking to patients about the "biologic treatment" rather than when or how the specific product came to market, which could be especially beneficial with patients who are newly starting therapy. For those patients who are already receiving therapy and would be switching to a biosimilar, a clinician could reference positive outcomes from studies evaluating switching or they could share testimonials from other patients who successfully switched-possibly a more impactful approach.

Additionally, participants noted that, for patients, the messaging must be relevant and individualized to their specific condition and situation; for example, as described above, messaging for a patient who is naive to the particular biologic therapy should be different from that for a patient who might be switching from a reference product to a biosimilar. It should also describe what changes they might 
expect to their daily life or treatment experience and how the biosimilar might affect affordability and whether they will see out-of-pocket savings. For many patients in the current environment, it will be critical to address public concerns about trust in regulatory agencies and to share appropriate information about their rigorous scrutiny of the safety and effectiveness of medications.

Related to information and messaging, participants considered education to be an opportunity to increase biosimilar adoption in the United States. They recommended building positive, evidence-based communication and educational materials around biosimilar adoption, differentiated to meet the needs of various audiences including patients, prescribers/providers, and payers. They believed it is important to have biosimilar-related continuing education or recertification programs for health care providers and to incorporate biosimilar education into health sciences curricula. Participants also suggested amplifying existing resources such as data, technology, advanced analytics, and FDA educational resources that can help clarify some of the complexities around biosimilars.

Participants discussed in depth the need to rebuild public confidence in science and the US drug approval process through education and positive messaging, particularly as they pertain to biosimilars. Strategies offered included patient testimonials of successful treatment with a biosimilar (as already mentioned) and consensus statements from trusted sources or organizations. Participants also encouraged sharing learning across disease states, stakeholder groups, and the globe, with the goal of collating and disseminating best practices from areas where biosimilar uptake efforts have succeeded. For instance, oncology was noted as a therapeutic area that has had relative success with biosimilar adoption and could be used as a model for other areas.

RWE was also seen as having a particularly important role in many of the aforementioned efforts. Specifically, collecting RWE and postmarketing surveillance data related to adverse events and side effects, switching of productsboth from a reference product to a biosimilar and from one biosimilar to another-persistence of biosimilar therapy, true differences in patient out-of-pocket costs and other economics, and utilization of biosimilars for off-label indications were all offered as areas for further research.

Participants also proposed organizational strategies to promote biosimilar adoption. Two examples were having a biosimilar champion with a dedicated focus to increase biosimilar utilization and using generic nomenclature to reduce confusion. Another example was for health plans/PBMs to initiate formulary changes, utilization management, and provider incentive programs or enhanced reimbursement that especially focus on treatment-naive patients, for whom biosimilar adoption may be more straightforward.

Participants identified legislation and regulations related to biosimilars as another area of opportunity. For instance, as the biosimilar marketplace grows, the experience gained with these products can be expected to spur the generation of more evidence and increase comfort around prescribing them; therefore, it is important to continue to combat attempts to unfairly delay competition and to reduce barriers that may keep biosimilars from gaining traction in the market. Reforming Medicare policies to promote biosimilar use via differential reimbursement under Part B and a biosimilar tier under Part D was an additional example, as were supporting greater public transparency on the pricing of biosimilars and reference biologics, and developing guidance to clarify existing legislative language.

\section{Conclusions}

Despite an established approval pathway and the possibility of significant savings to the US health care system, uncertainties and resistance remain around the use of biosimilars. During the course of this 2-day multistakeholder forum, participants discussed the challenges and barriers that contribute to this resistance; these include approval-related nuances and other regulatory considerations; general information about biosimilars and a lack of consistent, positive messaging; clinical and administrative barriers; and economic complexities and variation. Areas of opportunity highlighted by participants included demystifying information and developing strong, positive messaging around biologics and biosimilars; improving confidence in biosimilars by using tools such as education and RWE; implementing organizational strategies to ease biosimilar adoption; and updating legislation and regulation to reduce barriers related to biosimilars.

\section{DISCLOSURES}

This forum was sponsored by Amgen, the Association for Accessible Medicines, Boehringer Ingelheim, Fresenius Kabi, Johnson \& Johnson, Novo Nordisk, Pfizer, Sandoz, and Takeda. These proceedings were prepared as a summary of the forum to represent common themes; they are not necessarily endorsed by all attendees, nor should they be construed as reflecting group consensus. 


\section{ACKNOWLEDGMENTS}

The AMCP Partnership Forum was moderated by Clifford Goodman, PhD. This document was written by Bridget Flavin, PharmD, Founder, Connected Content, Ltd. Connected Content received payment from AMCP for the preparation of this manuscript, as well as from Avalere, Happe Consulting, Humana, Prime Education, PTCE (Pharmacy Times Continuing Education), University of Florida, and Xcenda. Flavin is also an employee of IngenioRx.

\section{REFERENCES}

1. US Food and Drug Administration. Biosimilar and interchangeable products. Updated October 23, 2017. Accessed June 1, 2021. https:// www.fda.gov/drugs/biosimilars/ biosimilar-and-interchangeable-products
2. US Food and Drug Administration. Abbreviated new drug application (ANDA). Updated May 22, 2019. Accessed June 12, 2021. https://www. fda.gov/drugs/types-applications/ abbreviated-new-drug-application-anda

3. US Food and Drug Administration. Biosimilars action plan: balancing innovation and competition. July 2018. Accessed June 6, 2021. https://www.fda.gov/ media/114574/download

4. US Food and Drug Administration. Biosimilars. Updated July 28, 2021. Accessed June 12, 2021. https:// www.fda.gov/drugs/therapeutic-biologics-applications-bla/ biosimilars
5. US Food and Drug Administration. Biosimilar development, review, and approval. Updated October 20, 2017. Accessed June 12, 2021. https://www.fda. gov/drugs/biosimilars/biosimilar-development-review-and-approval

6. Einchenbrenner PJ. Managed care stakeholders' opinions: strategies and barriers to biosimilar adoption. Data presented at: AMCP Virtual Partnership Forum; December 15, 2020.

7. Greene L, Singh RM, Carden MJ, Pardo CO, Lichtenstein GR. Strategies for overcoming barriers to adopting biosimilars and achieving goals of the Biologics Price Competition and Innovation Act: a survey of managed care and specialty pharmacy professionals. J Manag Care Spec Pharm. 2019;25(8):904-912. doi:10.18553/ jmcp.2019.18412 